\title{
Keeping Immunisation Coverage Targets in Perspective
}

\author{
David W. Brown*
}

United Nations Children's Fund, Three United Nations Plaza, New York 10017, USA

\begin{abstract}
Immunisation coverage targets have had a central role in global immunisation efforts. There is a growing focus on achievement of immunisation coverage targets by national immunisation programmes, particularly where targets are included as part of performance based funding arrangements. However, immunisation coverage targets should not become a distraction to national immunisation programme managers whereby short run forces to lead to a shift or abandonment of technically appropriate and sustainable activities in a country.
\end{abstract}

Keywords: Immunization programs, goals, program evaluation.

During recent visits to national immunisation programmes, I have been disturbed to hear pointed language focused on achievement of immunisation coverage targets and thresholds for the purposes of receiving new or continued donor financial support. Targets, or measureable goals, are a useful tool for assessing programme performance achieved as compared to expected performance. Targets are important to direct and focus programme activities, and can provide an organizing as well as a unifying principle for programmes. Moreover, they can enhance opportunities for a common dialogue within a national programme as well as across programmes in different countries. However, targets should not become the be-all and end-all or serve as a distraction whereby programmes allow short run forces to lead to a shift or abandonment of technically appropriate and sustainable activities. Unfortunately, this appears to be the case for some national immunisation programmes.

Coverage targets have historically played a central role within global immunisation. Of course, high levels of immunisation coverage are important to prevent and control vaccine-preventable diseases, and their measurement is used to monitor programs and to detect changes in the impact of programmes. The Expanded Programme on Immunisation (EPI), launched by the World Health Assembly in 1974, established a worldwide goal in 1977 of $80 \%$ coverage by 1990 for vaccines against tuberculosis, polio, diphtheria, pertussis, tetanus, and measles. A similar declaration was made for Universal Childhood Immunisation (UCI) in 1985 by the World Health Organization and United Nations Children's Fund. And, more recently the Global Immunisation Vision and Strategy (GIVS), a framework for protecting children from vaccine preventable childhood diseases, calls for raising global immunisation coverage to at least $90 \%$ by 2010 and sustaining these levels through at least 2015 [1]. Perhaps because immunisation coverage is considered a sensitive indicator of the health of a population

*Address correspondence to this author at the United Nations Children's Fund, Three United Nations Plaza, New York 10017 USA;

Tel: +1.212.303.79.88; E-mail: dbrown @ unicef.org and the capacity of a health system to deliver essential services [2], national immunisation coverage, namely the proportion of children immunized with three doses of diphtheria-tetanus-pertussis vaccine (DTP3) during the first year of life, has also been used as a performance indicator for the determination of funding eligibility for immunisation programmes (e.g., the Global Alliance for Vaccines and Immunization (GAVI), now the GAVI Alliance) and development more broadly (e.g., the Millennium Challenge Corporation).

Although concern for the use of immunisation coverage targets is not new, the tone behind discussions of the need to meet targets has recently become more fervent and the focus on them by some Ministries of Health has become consuming. And, from the country's perspective, concern for reaching coverage targets is completely rational, particularly given their use within performance-based funding arrangements - a relatively new phenomenon that may lead countries to choose short-term programme activities rather than take on the much needed long-term service and delivery strengthening and sustainability work. Such experiences seem reason enough to stop and ask whether appropriate direction and commentary is being provided to colleagues in national immunisation programmes or whether the global health and development community is somehow misguiding them. That is to say, to what extent does an over emphasis on coverage targets compromise the need to ensure quality and sustainability of national immunisation programmes in the long-run by reinforcing ineffective practices used to meet short-term objectives? Are the differences between programmes designed to achieve short-term, rapid immunisation coverage targets and those focused on longterm sustainable approaches recognized? I would like to think "yes", unfortunately given current focus and the use of immunisation coverage targets within performance-based funding arrangements, the answer may be to the contrary in some places.

International non-governmental agencies, philanthropic donors and other partners involved in global immunisation have an important role play in raising awareness that an emphasis on coverage targets may compromise the need to ensure quality and sustainability of national immunisation 
programmes. This is not to say that the use of targets should be abandoned; rather, more should be done to keep them in perspective. I am hopeful that the ongoing planning efforts for the so-called Decade of Vaccines [3], one of the largest single commitments in global health to-date, will alleviate the intense pressure to achieve coverage targets in the shortrun in lieu of strategies that are technically appropriate and that would yield sustainable levels of immunisation coverage, albeit below traditional targets as the case may be.

\section{DISCLAIMER}

The opinions expressed herein are those of the author alone and do not necessary reflect those of the United Nations Children's Fund.

\section{CONFLICT OF INTEREST}

None to declare.

\section{REFERENCES}

[1] Bilous J, Eggers R, Gasse F, et al. A new global immunisation vision and strategy. Lancet 2006, 367: 1464-6.

[2] Bos E, Batson A. Using immunization coverage rates for monitoring health sector performance. Measurement and interpretations issues. World Bank HNP Discussion Papers 2000

[3] Gates Foundation's decade of vaccines. Lancet Infect Dis 2010, 10: 139.

Received: January 19, 2011

Revised: January 25, 2011

Accepted: January 25, 2011

(C) David W. Brown; Licensee Bentham Open.

This is an open access article licensed under the terms of the Creative Commons Attribution Non-Commercial License (http://creativecommons.org/licenses/ by-nc/3.0/) which permits unrestricted, non-commercial use, distribution and reproduction in any medium, provided the work is properly cited. 